\title{
Observaciones sobre robo primario de néctar de Vespula vulgaris L., 1758 (Hymenoptera: Vespidae) en flores de Embothrium coccineum (J. R. Forst. \& G. Forst., 1775) (Proteaceae) en el sur de chile $\left(55^{\circ} \mathrm{S}\right)$
}

\author{
Observations of primary nectar robbing by Vespula vulgaris L., 1758 \\ (Hymenoptera: vespidae) on Embothrium coccineum (J. R. Forst. \& \\ G. Forst., 1775) (Proteaceae) flowers in southern Chile $\left(55^{\circ} \mathrm{S}\right)$
}

Javier Rendoll Cárcamo ${ }^{1,2}$, Tamara Contador ${ }^{1,2}$, Luna Menares Zúñiga ${ }^{3}$

El movimiento y transporte de especies mediado por humanos ha facilitado la presencia de especies exóticas en casi todos los ecosistemas. Algunas de estas especies se encuentran estrechamente asociadas a los entornos $y$ medios de transporte humanos, usualmente llegando a nuevas regiones sin ser detectadas por protocolos de inspección (Beggs et al. 2011, Work et al. 2005). Los Hymenoptera sociales, en particular especies de la familia Vespidae, suelen ser introducidos en nuevas localidades de esta manera (Beggs et al. 2011). Varias características convierten a los véspidos sociales en excelentes invasores; tienen alta capacidad de dispersión, alta tasa de reproducción, dietas generalistas, pueden utilizar una amplia gama de hábitats y explotar los recursos de una manera más eficiente que otros grupos de insectos (Moller, 1996). Además, sólo necesitan una reina fecundada para iniciar una nueva población (Beggs et al. 2011).

La avispa común Vespula vulgaris L., 1758 es una especie de origen Paleártico documentada como invasora en varios países del mundo (e.g. Australia, Nueva Zelanda, Islandia, Argentina, Chile) (Beggs et al. 2011). En América del Sur fue reportada por primera vez en Argentina el año 2010 (Masciocchi et al. 2010), y tres años después en Chile (Barrera-Medina \& Vidal, 2013). Sin embargo, dada sus similitudes morfológicas y conductuales con V. germanica F., 1793, detectada en Chile en 1975 (Peña et al. 1975), su presencia pudo haber pasado desapercibida. El primer reporte de V. vulgaris fue en el año 2015 en isla Navarino $\left(55^{\circ} \mathrm{S}\right)$, en el extremo austral de Chile (Rendoll et al. 2016), sin embargo las primeras referencias sobre avistamientos del género datan de 2012 (Sola et al. 2015). Estas avispas tienen un amplio impacto sobre la biodiversidad y el funcionamiento de los ecosistemas (Beggs et al 2011), siendo capaces de utilizar y explotar todos los recursos disponibles en sus nuevos ambientes, incluyendo carbohidratos, proteínas, agua y pulpa de madera para la construcción de sus nidos (Harris, 1991, Sackmann et al. 2000).

Los ladrones primarios de néctar, a diferencia de los visitantes florales legítimos, hacen agujeros en las flores (perforando, mordiendo o cortando los tubos florales) para obtener el néctar de una manera más directa (Inouye, 1980, Irwin et al. 2010). Las hormigas, abejas (abejorros, abejas carpinteras, abejas sin aguijón), y avispas, son los ladrones primarios más comunes (Barrows, 1980, Irwin et al. 2010). Casi todas las plantas de flores tubulares o con receptáculos de néctar experimentan este tipo de robo (Barrows, 1980). En el presente trabajo documentamos el robo de néctar de la avispa introducida $V$. vulgaris sobre

\footnotetext{
1 Laboratorio de Ecología Dulceacuícola Wankara, Programa de Conservación Biocultural Subantártica, Universidad de Magallanes, Puerto Williams, Chile. $\bowtie$ javier.rendoll@gmail.com

2 Instituto de Ecología y Biodiversidad, Departamento de Ciencias Ecológicas, Facultad de Ciencias, Universidad de Chile, Santiago, Chile.

3 Pontificia Universidad Católica de Chile, Santiago, Chile.
} 


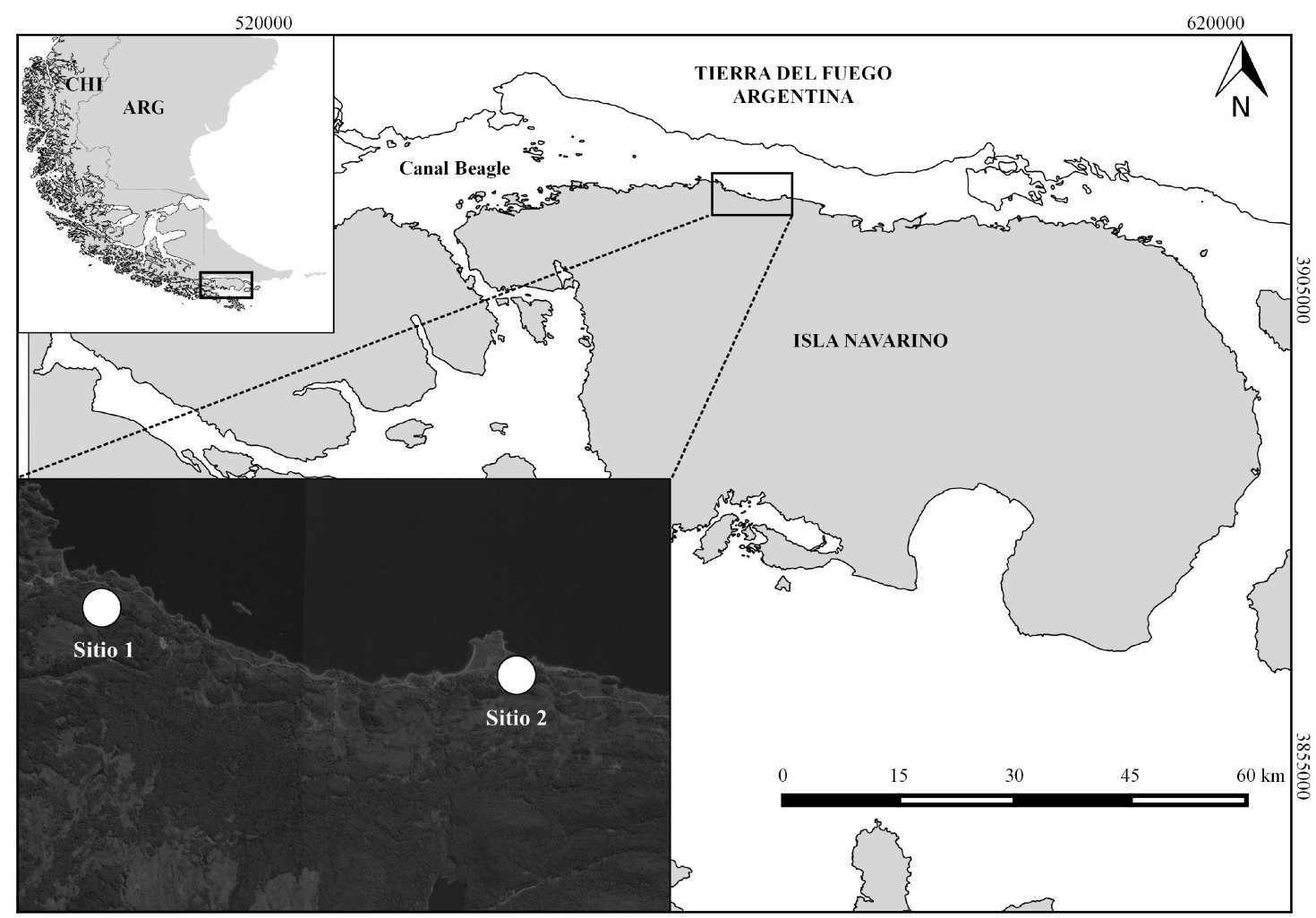

Fig. 1. Área de estudio en la costa Norte de isla Navarino, al sur de Sudamérica. Los círculos blancos representan los sitios muestreados. ARG = Argentina, $\mathrm{CH}=$ Chile. Proyección WGS 84/UTM 19S, elaboración propia, QGIS 2.8.2 Wien.

flores de un árbol nativo (Embothrium coccineum J. R. Forst. \& G. Forst., 1775) en isla Navarino.

No todos los visitantes florales son polinizadores, y algunos de estos se alimentan del néctar sin proveer el servicio de polinización. El robo de néctar puede tener diversas consecuencias, negativas o positivas, tanto en las plantas visitadas como en la interacción entre plantas y sus visitantes polinizadores nativos (Maloof \& Inouye, 2000). A pesar de haberse registrado solo hace algunos años en la isla Navarino, $V$. vulgaris se encuentra presente a lo largo de toda su costa Norte (datos no publicados). Sin embargo, poco se conoce sobre su interacción con la flora y fauna nativa.

Con el objetivo de cuantificar el robo de néctar por parte de avispas en isla Navarino, durante los meses de enero y febrero de 2017 realizamos monitoreos sobre la distribución de $V$. vulgaris y $V$. germanica a lo largo de la costa norte de la isla (Fig. 1). El área de estudio es parte de la ecorregión subantártica de Magallanes, compuesta principalmente por bosques de Nothofagus, matrices de humedales y turbales, conocidas como el complejo de tundra de Magallanes (Pisano, 1977). Las observaciones se focalizaron en un área de matorral secundario, post incendio forestal, dominado por Embothrium coccineum (Rozzi \& Jiménez, 2014). Embothrium coccineum es un árbol siempre verde de pequeño tamaño con inflorescencias rojas arregladas en racimos de 10 a 40 flores tubulares (Smith-Ramírez \& Armesto, 2003).

Los monitoreos consistieron en estaciones de observación de 10 minutos por árbol de E. coccineum en el que avispas eran observadas. Para determinar cómo las avispas acceden al néctar colectamos cada inflorescencia en la que las avispas se alimentaban. Cada sitio fue visitado durante el mismo día y dos veces por semana, principalmente durante febrero, mes con mayor abundancia 


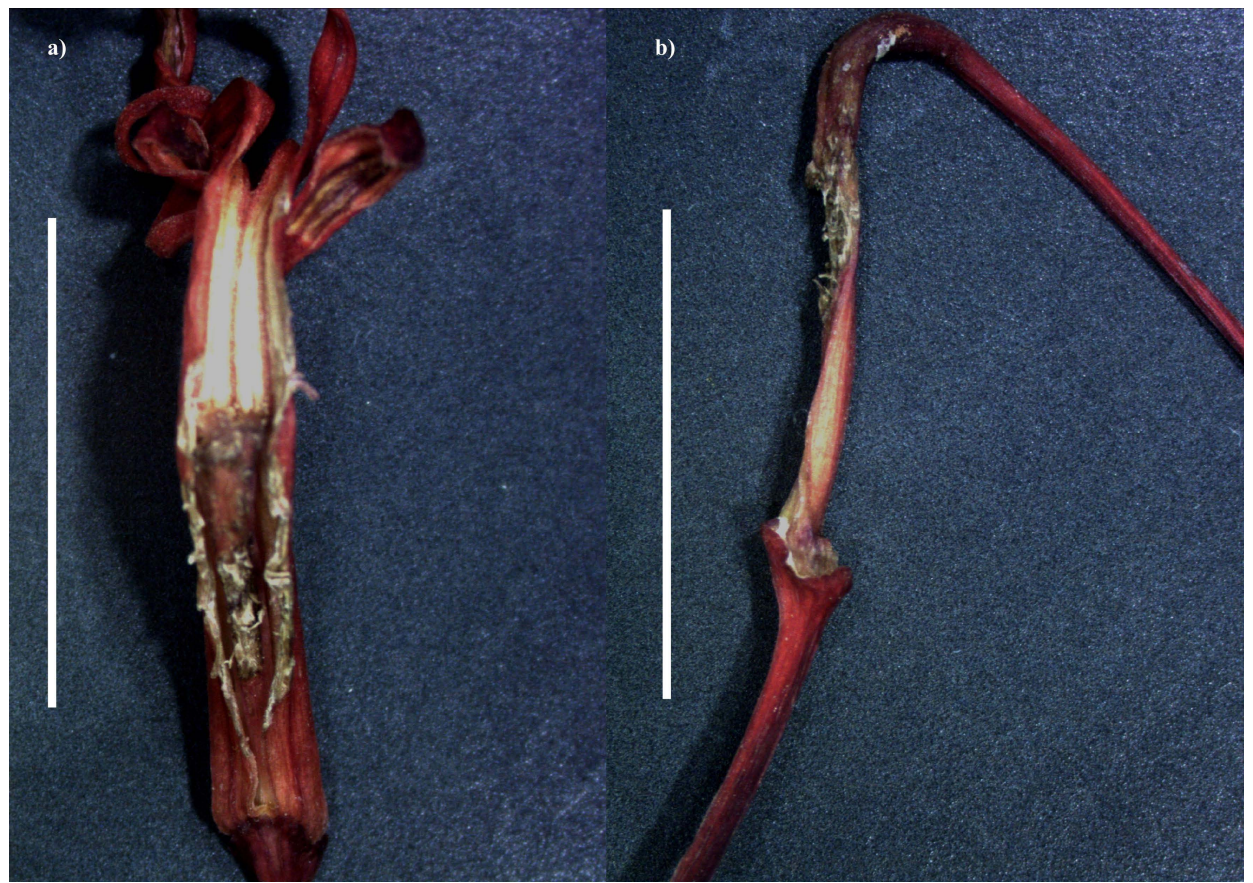

Fig. 2. Marcas y daño que realiza Vespula vulgaris sobre las flores de E. coccineum, a) agujero realizado en la corola por la mordida de $V$. vulgaris, y b) filamento con marcas de mordida. La línea blanca representa una medida de $10 \mathrm{~mm}$.

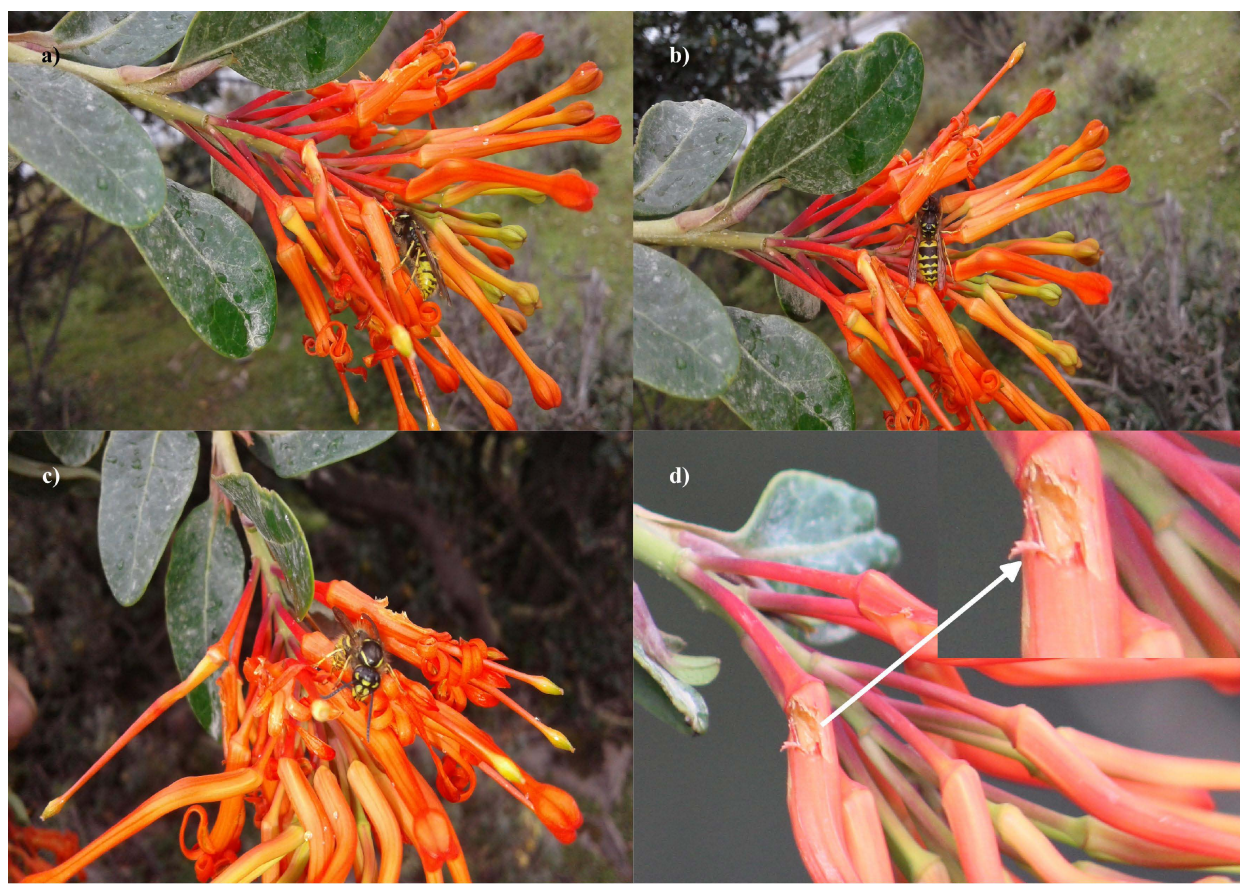

Fig. 3. a, b, c) Vespula vulgaris mordiendo la base de las corolas de las flores de E. coccineum, d) Flor de E. coccineum con marcas de mordida. La flecha blanca indica el área mordida, aumentada 2.5 veces a la derecha. Fotografía tomada en la costa Norte de isla Navarino en un ambiente de matorral (Sitio 1). 
y actividad de individuos de estas avispas. Los individuos de Vespula spp. fueron colectados con red entomológica, y para su identificación se siguió a Dvorak y Roberts (2006) y la adaptación de Barrera y Vidal (2013).

Se determinó que todos los especímenes colectados sobre las inflorescencias de $E$. coccineum pertenecen a obreras de $V$. vulgaris $(\mathrm{n}=27)$. Adicionalmente, colectamos un total de 78 inflorescencias en 30 árboles de E. coccineum, todas con marcas de daño realizado por $V$. vulgaris. En isla Navarino, esta avispa consigue robar néctar haciendo agujeros con sus mandíbulas en la base de las corolas de las flores, similar a los abejorros y abejas sin aguijón (Fig. 2,3), actuando como un ladrón primario de néctar. Un estudio previo, en el área nativa de distribución de la especie, Reino Unido, reporta a V. vulgaris como un ladrón de néctar secundario, aprovechando los agujeros realizados por Bombus terrestris (Apidae) (Corbet et al. 1981). Esta discrepancia entre el robo de néctar en su hábitat nativo y donde ha sido introducida puede estar relacionada a un evento de introducción anterior al de B. terrestris. Otros véspidos ladrones de néctar documentados incluyen a Epipona guerini (Polistinae) en Costa Rica (Young, 1980), V. maculifrons (Vespinae) en los Estados Unidos (Zimmerman \& Cook, 1985), y Ancistrocerus oviventris (Eumeninae) en Alemania (Haeseler, 1997).

En Chile, se conocen al menos 20 especies animales que vistan las flores de E. coccineum, principalmente aves e insectos (Rovere \& Chalcoff, 2010). En la isla Navarino, la cachaña Enicognathus ferrugineus y el cometocino patagónico Phrygilus patagonicus se alimentan de las flores y semillas de E. coccineum, mientras que el picaflor chico Sephanoides sephaniodes y el fío fío Elaenia albiceps chilensis actúan como polinizadores vertebrados (Rovere \& Chalcoff, 2010).

Vespula vulgaris, así como otros ladrones de néctar, pueden dañar directamente los órganos reproductivos de las plantas con potenciales consecuencias negativas en su fitness (McDade \& Kinsman, 1980; Maloof \& Inouye, 2000). Por otro lado, pueden alterar el comportamiento de los polinizadores mediante competencia por interferencia, limitando la cantidad de néctar y/o el atractivo floral, lo que podría conducir a cambios a largo plazo en los rasgos florales (Roubik, 1982; Irwin et al. 2010). Los himenópteros sociales, en particular los véspidos, son conocidos por su agresividad territorial y durante el forrajeo (Rust \& Su 2012). La abeja sin aguijón Trigona ferricauda persigue picaflores lejos de las flores a las que roba néctar en Panamá (Roubik, 1982). En isla Navarino, $\mathrm{V}$. vulgaris persigue y aleja a paseriformes de los arbustos con bayas de Berberis microphylla (obs. pers.). Dado su agresivo comportamiento es probable que $V$. vulgaris persiga y aleje visitantes de las flores de E. coccineum.

Los resultados de este estudio muestran la amenaza potencial a la flora nativa de isla Navarino dada la presencia de $V$. vulgaris se recomienda implementar medidas de control con el objetivo de disminuir el tamaño poblacional de Vespula spp., y prevenir una mayor propagación. Esperamos que esta nota despierte la atención de la comunidad científica local, para incrementar los estudios con la finalidad de entender los efectos directos sobre el fitness de las poblaciones de plantas e insectos nativos de los que se alimentan.

\section{AGRADECIMIENTOS}

Agradecemos a Lorena Saavedra, Fernando Cárdenas y Miguel Gallardo por su ayuda en terreno, a Nancyrose Houston, Rocío Jara y Ramiro D. Crego por comentar en las primeras versiones del manuscrito. Adicionalmente, agradecemos las valiosas sugerencias de dos revisores anónimos que contribuyeron a mejorar este manuscrito. Este trabajo es parte de la iniciativa "Niche modelling, invasion stage and habitat preferences of alien Vespula wasps (Hymenoptera: Vespidae) in Navarino island $\left(55^{\circ} \mathrm{S}\right)$, southern Chile" del Laboratorio de Ecología Dulceacuícola Wankara.

\section{LITERATURA CITADA}

Barrera-Medina, R., \& Vidal, C. (2013). Primer reporte de Vespula vulgaris (Linnaeus, 1758) (Hymenoptera: Vespidae) en Chile. Boletín de la Sociedad Entomológica Aragonesa, 52, 277-278.

Barrows, E. M. (1980) Robbing of exotic plants by introduced carpenter and honey bees in Hawaii with comparative notes. Biotropica, 
12, 23-29.

Beggs, J. R., Brockerhoff, E. G., Corley, J. C., Kenis, M., Masciocchi, M., Muller, F., Rome, Q., \& Villemant, C. (2011). Ecological effects and management of invasive alien Vespidae. BioControl, 56(4), 505-526.

Corbet, S. A., Cuthill, I., Fallows, M., Harrison, T., \& Hartley, G. (1981) Why do nectarforaging bees and wasps work upwards on inflorescences?. Oecologia, 51, 79-83.

Dvořák, L., \& Roberts, S. P. (2006). Key to the paper and social wasps of Central Europe (Hymenoptera: Vespidae). Acta Entomologica Musei Nationalis Pragae, 46, 221-244.

Haeseler, V. (1997) Ancistrocerus oviventris (Wesmael 1836), another primary nectar robbing solitary wasp (Hymenoptera: Vespoidea: Eumenidae). FaunistischOekologische Mitteilungen, 7, 259-66

Harris, R. J. (1991). Diet of the wasps Vespula vulgaris and $V$. germanica in honeydew beech forest of the South Island, New Zealand. New Zealand Journal of Zoology, 18, 159-169.

Inouye, D. W. (1980). The terminology of floral larceny. Ecology, 61, 1251-1253.

Irwin, R.E., Bronstein, J.L., Manson, J.S., \& Richardson, L. (2010) Nectar robbing: ecological and evolutionary perspectives. Annual Review of Ecology, Evolution, and Systematics, 41, 271-292.

Maloof, J. E., \& Inouye, D. W. (2000). Are nectar robbers cheaters or mutualists? Ecology, 81, 2651-61.

McDade, L. A., \& Kinsman, S. (1980). The impact of floral parasitism in two neotropical hummingbird-pollinated plant species. Evolution, 34, 944-58.

Masciocchi, M., Beggs, J. R., Carpenter, J. M., \& Corley, J. C. (2010). Primer registro de Vespula vulgaris (Hymenoptera: Vespidae) en la Argentina. Revista de la Sociedad Entomológica Argentina, 69, 267-270.

Moller, H. (1996). Lessons for invasion theory from social insects. Biological Conservation, 78, 125-142.

Peña, L., Pérez de Arce, R., \& Cartagena, L. (1975).
La presencia de Vespula maculifrons (Buysson) (Hymenoptera: Vespidae) en Chile. Revista Chilena de Entomología, 9, 167-168.

Pisano, E. (1977). Fitogeografía de FuegoPatagonia. Anales del Instituto de la Patagonia chilena Serie Ciencias Naturales, 8, 121-250.

Rendoll, J., Contador, T., Crego, R. D., Jordan, N. I., Schüttler, E., Gañán, M., Jiménez, J.,.. \& Kennedy, J.H. (2016). Primer registro de Vespula vulgaris (Linnaeus 1758) (Hymenoptera: Vespidae) en la isla Navarino, Chile. Gayana, 80, 133-136.

Roubik, D. W. (1982). The ecological impact of nectar-robbing bees and pollinating hummingbirds on a tropical shrub. Ecology, 63, 354-360.

Rovere, A. E., \& Chalcoff, V. R. (2010). Embothrium coccineum: JR Forst. et G. Forst. Kurtziana, 35, 23-33.

Rozzi, R., \& Jiménez, J. E. (2014). Magellanic sub-Antarctic ornithology: first decade of bird studies at the Omora Ethnobotanical Park, Cape Horn Biosphere Reserve, Chile. University of North Texas press, Universidad de Magallanes, Denton, Punta Arenas.

Rust, M. K., \& Su, N. Y. (2012). Managing of social insects of urban importance. Annual Review of Entomology, 56, 355-375.

Sackmann, P., D'Adamo, P., Rabinovich, M., \& Corley, J. C. (2000) Arthropod prey foraged by the German wasp (Vespula germanica) in NW Patagonia, Argentina. New Zealand Entomologist, 23, 55-59.

Smith-Ramirez, C., \& Armesto, J. J. (2003) Foraging behavior of bird pollinators on Embothrium coccineum (Proteaceae) trees in forest fragments and pastures in southern Chile. Austral Ecology, 28, 53-60.

Sola, F. J., Valenzuela, A. E. J., Anderson, C. B., Martínez Pastur, G., \& Lencinas, M. V. (2015). Reciente invasión del Archipiélago de Tierra del Fuego por la avispa Vespula germanica (Hymenoptera: Vespidae). Revista de la Sociedad Entomológica Argentina, 74(3-4), 197-202.

Work, T. T., McCullough, D. G., Cavey, J. 
F., \& Komsa, R. (2005). Arrival rate of nonindigenous insect species into the United States through foreign trade. Biological Invasions, 7, 323-332.

Young, H. J., \& Stanton, M. L. (1990). Influences of floral variation on pollen removal and seed production in wild radish. Ecology, 71(2), 536-547.

Zimmerman, M., \& Cook, S. (1985). Pollinator foraging, experimental nectar-robbing and plant fitness in Impatiens capensis. American Midland Naturalist, 84-91. 